\title{
Role of diet in epigenetics: a review
}

\begin{abstract}
Epigenetics refers to the mechanisms that alter gene expression without altering the primary DNA sequence. The epigenetic modifications may be due to cytosine base methylation in DNA or by post-translational biochemical modifications of histones, the core proteins of nucleosomes. Epigenetic mechanisms are heritable and reversible. Epigenetic modifications can occur in response to environmental stimuli, of which the most important one is diet. Nutrients can modify physiologic processes through epigenetic mechanisms. The dietary nutrients enter metabolic pathways and get modified in to simpler forms for the body to utilize. One carbon cycle is such a unique pathway which produces methyl groups to silence the genes. Nutrients like methionine, Vitamin $B_{12}$, folic acid, and Vitamin $B_{6}$, are the key components of this pathway. These methyl-donating nutrients in the diet can alter gene expression. The knowledge regarding nutritional epigenetics is still limited. As Epigenetics is reversible in nature, it is now considered as an attractive field of nutritional intervention. In the future, we need to investigate on more nutrients or bioactive food compounds to find the ones that can improve human health.
\end{abstract}

Keywords: epigenetics, diet, nutrients, one carbon cycle
Volume 7 Issue 3 - 2018

\author{
Abhina Mohanan, Raji Kanakkaparambil \\ Department of Veterinary Physiology, College of Veterinary and \\ Animal Sciences, India
}

Correspondence: Abhina Mohanan, Department of Veterinary Physiology, College of Veterinary and Animal Sciences, Mannuthy, Thrissur, Kerala-68065I, India, Tel +91-9400973986,

Email drabhinavet।31।@gmail.com

Received: December 23, 2016 | Published: June 25, 2018
Abbreviations: $\mathrm{CpG}$, cytosine phosphate guanine; IGF-2 DMR: Insulin like growth factor-2 differentially methylated region

\section{Introduction}

The word epigenetic seems to be a new word for us, but it has been studied a long while ago. The name epigenetics was coined by Conrad Waddington in 1942. "Epi", in Greek, means "above" or "beyond"., Epigenetics was explained as a multifaceted developmental process connecting genotype and phenotype. ${ }^{3}$ DNA is the genetic blueprint to create a living organism, while the epigenetic information provides the message on how, where and when the genetic material should be used. ${ }^{4}$ Nutrients can modify physiologic processes through epigenetic mechanisms that alter the gene expression. The link between nutrition and epigenetics can be elucidated by metabolic pathways especially the one carbon metabolism. Nutrients from our food are modified in one carbon pathway that extracts methyl groups and then attaches them to our DNA or proteins. One carbon metabolism involving the folate and methionine cycle, integrates carbon units from amino acids and generates substrates for methylation of nucleic acid and protein. Nutritional deficiencies in folate, choline, methionine, vitamins $\mathrm{B}_{6}$ and $\mathrm{B}_{12}$ perturb the complex regulatory network that maintains one-carbon metabolism. Polymorphisms in genes encoding the enzymes involved in metabolic pathways can also alter one carbon metabolism. Asepigenetics is reversiblein nature, it is now considered as an attractive field of nutritional intervention. ${ }^{5}$ In this review, we are focusing mainly on the nutrients and metabolic pathways leading to epigenetic modification by DNA methylation.

\section{Epigenetic mechanisms affecting gene expression}

Nucleosomes, the fundamental repeating units of chromatin can be modified by many types of biochemical processes such as methylation, acetylation, phosphorylation, ubiquitylation, and sumoylation. ${ }^{6}$ The epigenetic modifications may be due to

i. cytosine base methylation in DNA ii. post-translational modifications of histone proteins

iii. Positioning of nucleosomes along the DNA.

Epigenetic modifications can regulate the state of chromatin into a euchromatic (highly active) or heterochromatic (completely silent) state. Epigenetic modification of DNA at $\mathrm{CpG}$ islands modifies cytosine base and opposes transcription in most cases. ${ }^{7}$ Promoter regions with $\mathrm{CpG}$ islands become methylated during development, which results in long-term transcriptional silencing and inactivation of X-chromosome and imprinted genes which are classic examples of naturally occurring $\mathrm{CpG}$ island methylation during development. ${ }^{7}$ In the current scenario, DNA demethylation mechanism is gaining importance as it is necessary for cellular processes during development stages. ${ }^{5,7}$ The importance of the DNA methylation is that it facilitates the silencing of the imprinted genes ${ }^{5}$ and ${ }^{9}$ mainly focused on the role of early life nutrition causing epigenetic modifications. DNA methyltransferases (Dnmts) are the enzymes those control DNA methylation and regulate gene expression patterns. Currently, five different Dnmts are known: Dnmt1, Dnmt2, Dnmt3a, Dnmt3b and DnmtL. Dnmt1 are the maintenance methyl transferase and Dnmt3a, $3 \mathrm{~b}$, and $\mathrm{L}$ are the de novo ones.

The histones are the major protein components of chromatin, and they have a significant role in gene regulation. The role of histone modifications in RNA synthesis has been very well reported. ${ }^{10}$ The five types of histones in chromatin are $\mathrm{H} 1 / \mathrm{H} 5, \mathrm{H} 2 \mathrm{~A}, \mathrm{H} 2 \mathrm{~B}, \mathrm{H} 3$, and $\mathrm{H} 4$, and together with DNA,they form the nucleosome, which forms the chromatin. ${ }^{11}$ It is suggested that their modification can result in both repressive and stimulatory effects on genomic functions in vivo. ${ }^{12,13}$ It has been reported that histone modifications can either activate or repress the genes based on the type of residues which are modified and also the type of modifications occurred. ${ }^{14}$ By definition, epigenetic changes are heritable changes, which can be reversed. ${ }^{15}$ This heritability is established during differentiation stage and through multiple cycles of cell division it is being maintained with same genetic information, and thus the identity of the cells do not change. ${ }^{14}$ 


\section{Role of diet in epigenetics}

Many scientists have studied the influential role of environment (mainly the diet) in changing the phenotype of an individual. The studies conducted in honey bees are one of the best examples to show the role of diet in epigenetics. ${ }^{16,17}$ Both fertile queens and sterile workers develop from the same ancestor, but they differ only because of the diet they are being fed. ${ }^{17}$ The genetically identical honeybee queen and workers get differentiated only because of the special diet called as 'royal jelly' fed to the larvae. ${ }^{18}$ In 1944 a famine, later named as Dutch Hunger Winter Famine, affected Netherlands. The environmental influence on the growth rate of that time (Barker hypothesis) was proposed which established that the men having lowest weights at early stages of development with poor nutrition had the highest death rates from cardiac diseases. ${ }^{19}$ If nutrition is not good enough during these stages, imperfections will be there in the growing person, even with the right genetic make-up, and this will make someone more prone to illness later in life. Studies were conducted in adult periconceptional individuals exposed to famine and found significant differences in IGF2 DMR methylation in adulthood. ${ }^{20}$ These findings demonstrate that even with the right genetic makeup, diet is one of the factors which can modify gene expressions and improper diet may be an etiology for many of adulthood diseases. The traditional concept of the role of early life nutrition in changing the epigenetic mechanisms causing disease condition has been substantiated. ${ }^{9}$ These data were the first to prove direct link between epigenetic modifications and diet that persist throughout life.



Figure I The methionine cycle.

\section{Acknowledgements}

None.

\section{Conflict of interest}

The author declares no conflict of interest for this manuscript.

\section{References}

1. M Brait, D Sidransky. Cancer epigenetics: above and beyond. Toxicol Mech Methods. 2011;21(4):275-288.

2. J Shulevitz. Why Fathers Really Matter. The New York Times. 2012.

3. CH Waddington. The epigenotype.1942. Int J Epidemiol. 2012;41(1):1013.
Epigenetic changes occur by various dietary nutrients, and some of the essential nutrients involved in methylation process are folate, Vitamin B12, methionine, choline and betaine. The link between nutrition and epigenetics can be elucidated by metabolic pathways especially the one-carbon metabolism. The methionine cycle (Figure 1 ) is central to one carbon metabolism. The universal methyl donor in the one-carbon cycle is S-adenosyl methionine (SAM). Methionine adenosyl transferase (MAT) converts methionine to SAM, and most of the SAM formed is used for transmethylation reactions including DNA and histone methylation. ${ }^{21}$ Homocysteine which is formed enters transulfation pathway to form cystathionine which require vitamin $\mathrm{B}_{6}$ as a co-factor. Alternatively, homocysteine is remethylated to methionine using the enzymes: methionine synthase (which requires folic acid as the methyl donor and vitamin $\mathrm{B}_{12}$ as a co-factor) or (b) betaine homocysteine methyl transferase (BHMT), for which betaine (tri-methyl glycine) acts as the methyl donor. ${ }^{22}$ Therefore, homocysteine levels in the blood are strongly influenced by diet, especially the B-complex vitamins. Choline also acts as methyl donor in the conversion of homocysteine to methionine. Therefore the one-carbon metabolism clearly explains the importance of diet in maintaining a proper epigenetic status of the individual. ${ }^{21-23}$ The effect of these components on DNA methylation has been reviewedin detail by Choi \& Friso. ${ }^{5}$ Studies on the role of various dietary factors, including micronutrients and non-nutrient dietary components such as polyphenols and genistein in epigenetic modifications were reviewed thoroughly. ${ }^{24}$

\section{Future Perspectives}

"You are what you eat"25 and also epigenetics prove that you are what your ancestors ate. ${ }^{26-28}$ Individual programmed traits which we inherit get altered by environmental factors including diet which may lead to diseases. Bacalini et al., ${ }^{15}$ has recently reviewed the inheritance pattern of epigenetics and the importance of environmental factors during different stages of development in utero. Recent discoveries have helped focus attention on the outstanding contributions of epigenetics in preventing diseases. As per Bacalini et al., ${ }^{15}$ as age advances, epigenetic modifications also are positively related to diet, physical exercise, and lifestyle habits. However, we are still in the infancy of such studies, and that much has to be done in order to unravel the complex relationship between epigenetics, nutrition and ageing. Limited knowledge on the effects of nutrients or bioactive food components on histone modifications or chromatin remodeling complexes paves the way for further investigation on nutrients effect to improve human health. ${ }^{9}$

4. KX Xue. Epigenetics promotes a new round of the genetic development. Yi Chuan. 2005;27(1):155-159.

5. S Choi, S Friso. Epigenetics: A New Bridge between Nutrition and Health. Adv. Nutr. 2010;1(1):8-16.

6. B Weinhold. Epigenetics: The Science of Change. Environ Health Perspect. 2006;114(3):A160-A167.

7. A Bird. DNA methylation patterns and epigenetic memory. Genes Dev. 2002;16(1):6-21.

8. E Li, C Beard, R Jaenisch. Role for DNA methylation in genomic imprinting. Nature.1993;366(6453):362-365.

9. KA Lillycrop, GC Burdge. Epigenetic mechanisms linking early nutrition to long term health. Best Pract Res Clin Endocrinol Metab. 2012;26(5):667-667. 
10. VG Allfrey, R Faulkner, AE Mirsky. Acetylation and methylation of histones and their possible role in the regulation of Rna synthesis. Proc Natl Acad Sci. 1964;51(5):786-794.

11. M Bhasin, EL Reinherz, PA Reche. Recognition and classification of histones using support vector machine. J Computational Biol. 2006;13(1):102-112.

12. PT Georgel, JC Hansen. Linker histone function in chromatin: Dual mechanisms of action. Biochem Cell Biol. 2001;79:313-316.

13. T Kouzarides. Chromatin modifications and their function. Cell. 2007;128(4):693-705

14. S Sharma, $\mathrm{T} \mathrm{K}$ Kelly, PA Jones. Epigenetics in cancer. Carcin 2010;31(1):27-36.

15. MG Bacalini, S Friso, F Olivieri, et al. Present and future of anti-ageing epigenetic diets. Mech Ageing Dev. 2014;136:101-115.

16. Y Wang, M Jorda, PL Jones, et al. Functional CpG Methylation System in a Social Insect. Science. 2006;314(5799):645-647.

17. R Kucharski, J Maleszka, S Foret, et al. Nutritional control of reproductive status in honeybees via DNA methylation. Science. 2008;319(5871):18271830 .

18. J Tautz. The buzz about bees. The biology of a super organism. Springer: 2008. p. 84-91.

19. DJP Barker, C Osmond, PD Winter, et al. Weight in infancy and death from ischaemic heart disease. Lancet. 1989;334(8663):577-580.
20. BT Heijmans, EW Tobi, AD Stein, et al. Persistent epigenetic differences associated with prenatal exposure to famine in humans. Proc Natl Acad Sci. 2008;105(44):17046-17049.

21. JM Mato, L Alvarez, P Ortiz. S-Adenosylmethionine synthesis: Molecular mechanisms and clinical implications. Pharmacol Ther. 1997;73(3):265280 .

22. Hoffer LJ. Homocysteine remethylation and trans-sulfuration. Metabol. 2004;53(11):1480-1483.

23. Smythies J. The Role of Abnormalities Related to the One Carbon Cycle in Depression and Schizophrenia. Neuroscience \& Medicine. 2012;3:101106.

24. JA McKay, JC Mathers. Diet induced epigenetic changes and their implications for health. Acta Physiol. 2011;202(2):103-118.

25. F Jacka. You are what you eat: how diet affects mental well-being. 2014.

26. Mercola. Falling for This Myth Could Give You Cancer. 2012.

27. E Ho, LM Beaver, DE Williams, et al. Dietary Factors and Epigenetic Regulation for Prostate Cancer Prevention. Adv Nutr. 2011;2(6):497-510.

28. R Kanakkaparambil. Methyl Metabolism and ovarian follicle development in sheep. 2008. 